\title{
Gallstone lleus: Diagnostic and Surgical Dilemma
}

\author{
Vincenzo Leone
}

\begin{abstract}
Background: The typical patient with Gallstone ileus is female, elderly, with concomitant medical diseases and high operative risk. This disease is becoming more common as a result of increase the aging population. Its diagnosis is difficult and early diagnosis could reduce the mortality. Nowadays the use of new imaging techniques can expedite the correct diagnosis, decreasing preoperative delay. However, controversy persists primarily in relation to surgical strategy.
\end{abstract}

Methods: We retrospectively reviewed the medical records of all patients with the diagnosis of intestinal obstruction between 1998 and 2011. We took into consideration concomitant medical conditions, previous history of cholelithiasis, operative risk (ASA score), preoperative findings, duration and type of surgery, post-operative outcome, 30-day mortality.

Results: The incidence was $0.66 \%$ (4 out of 601 cases of intestinal obstruction), $1 \%$ if we consider small bowel obstruction only (399 cases), $2.01 \%$ in patients over the age of 65 (199 out of 601) and $3.73 \%$ in patients over the age of 80 (107 out of 601$)$. In cases of small bowel obstruction resulting in impacted foreign body the incidence was $36.36 \%$. All patients were female with a mean age of 81.7 (range 75 - 86). The proper diagnosis prior to surgery was assessed in 2 out of 4 cases. In one case enterolithotomy was completed in one stage with cholecystectomy and closure of the fistula during acute surgery, while in 3 cases enterolithotomy alone was performed, 1 patient operated enterolithotomy alone died on the 14 th postoperative.

Conclusions: The history, clinical, and radiologic findings are often nonspecific suggesting only a small bowel obstruction. Nowadays the use of new imaging techniques, in combination with plain

Manuscript accepted for publication June 7, 2012

Department of General Surgery, St. Maria Nuova Hospital, Piazza St. Maria Nuova, 1 50122, Firenze, Italy. Email: vinc_leone@tin.it

doi: $10.4021 / \mathrm{jcs} 87 \mathrm{w}$ abdominal radiographs, can expedite the correct diagnosis in over $50 \%$ of cases decreasing preoperative delay. The type of intervention does not significantly influence post-operative morbidity and mortality rates. We believe to perform simple enterolithotomy as procedure of choice, while the one-stage procedure should be performed in patients with greater life expectancy, less operative risk and comorbidity.

Keywords: Gallstone ileus; Bilioenteric fistula; Enterolithotomy; Pneumobilia

\section{Introduction}

Gallstone ileus (GSI) is a rare mechanical intestinal obstruction caused by intraluminal impaction of one or more gallstones passed through bilioenteric fistula. GSI accounts for $1-3 \%$ of all mechanical intestinal obstruction and occurs mainly in elderly patients and it is becoming more common as a result of increase the aging population [1]. There is a female predominance (female to male ratio is $3.5-6: 1$ ). This disease is associated with a high mortality (7.7-15\%) and morbidity mainly due to the presence of concomitant medical conditions in elderly patients and non-specific symptoms resulting in delayed preoperative diagnosis [2]. Because of this, diagnosis is detected only at laparotomy in over $50 \%$ of cases [3]. Nowadays laparoscopic surgery and lithotripsy have been employed, but the open surgery is mostly performed. However, controversy persists primarily in relation to surgical strategy because of low incidence of this disease.

\section{Methods}

We retrospectively reviewed the medical records of all patients with the diagnosis of intestinal obstruction treated in our institution between 1998 and 2011. Records were retrieved using the ICD-9-CM coding system. In all patients we took into consideration concomitant medical conditions, previous history of cholelithiasys, operative risk assessment according to the American Society anaesthesiologists (ASA 


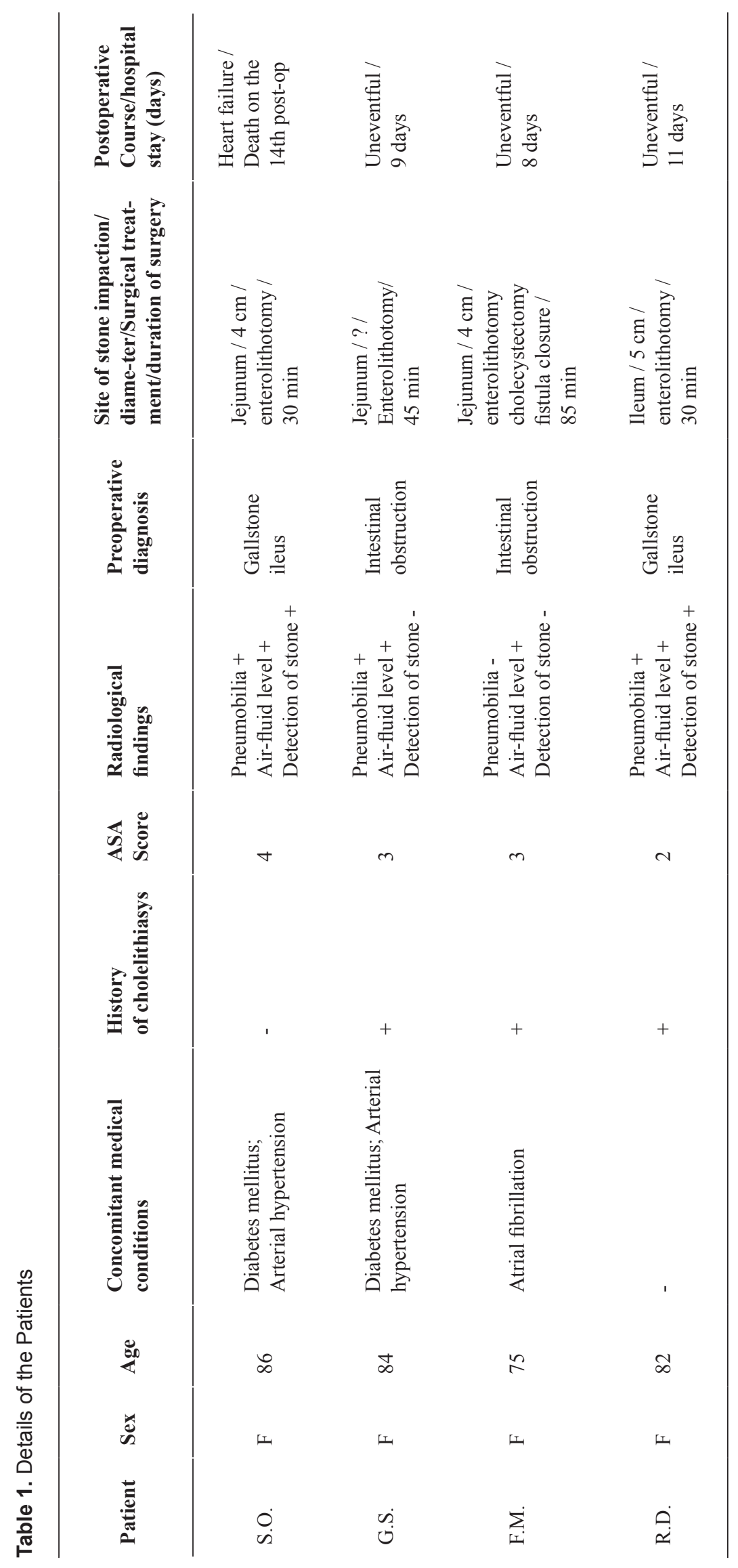




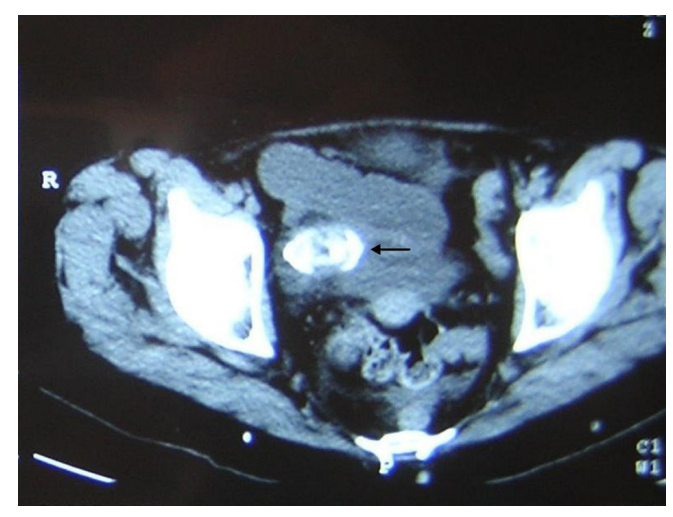

Figure 1. Abdominal tomography shows ectopic stone in the ileum.

score), preoperative findings, duration and type of surgery, post-operative outcome, 30-day mortality.

\section{Results}

We found a total of 601 patients with the diagnosis of intestinal obstruction, of these there were 4 cases of GSI. All patients were female with a mean age of 81.7 (range 75 86). The main details of patients are resumed in the Table 1. The incidence of GSI was $0.66 \%$ of operations for intestinal obstruction (4/601 cases), but if we consider small bowel obstruction only (399 cases), the incidence was $1 \%$. However, if we consider elderly patients with small bowel obstruction only, the incidence increases: $2.01 \%$ in patients over the age of 65 (199 out of 601 ) and $3.73 \%$ in patients over the age of

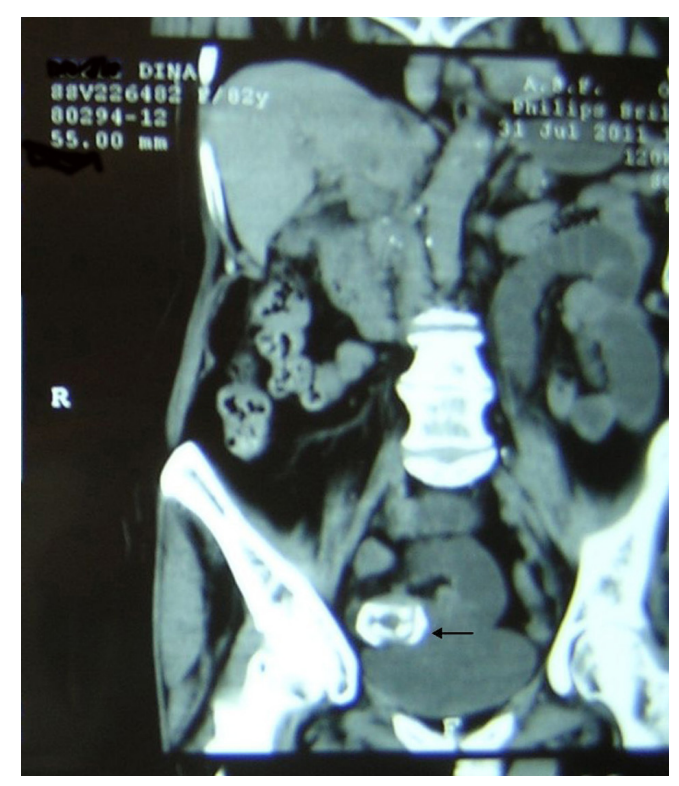

Figure 2. Tomographic image of the Rigler's triad.

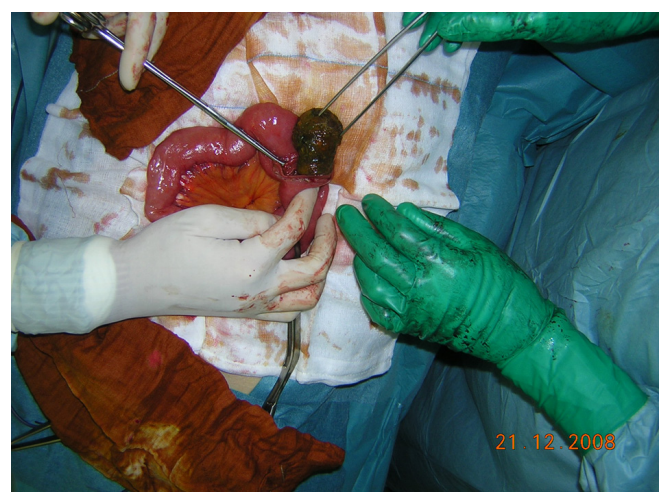

Figure 3. Enterotomy and extraction of the stone.

80 (107 out of 601). Finally, in cases of small bowel obstruction resulting in impacted foreign body (11 patients: 5 phytobezoar, 4 gallstone ileus, 2 dental prosthesis) the incidence was $36.36 \%$. All patients were admitted to emergency department with the diagnosis of intestinal obstruction. Median duration of symptoms before admission to the hospital was 1.7 days (range 1 - 3). On admission, three patients (75\%) had a known history of cholelithiasis, while none undergone previous biliary surgery. All patients were operated within 24 hours of admission to emergency department. The white blood count was higher $15000 / \mathrm{mL}$ in 3 out of 4 cases. Preoperative plain abdominal radiographic was nonspecific and inconclusive in all patients, showing only multiple intestinal air-fluid levels. Preoperative ultrasonographic evaluation revealed multiple gallstones in the gallbladder in all patients and pneumobilia in 2 out of 4 cases. The presence of ectopic stone in the ileum was not demonstrated by this examination. In 2 out of 4 cases (50\%) preoperative abdominal computed tomography (CT) revealed ectopic stones in the ileum (Fig. 1), pneumobilia and multiple intestinal air-fluid levels (Rigler's triad) (Fig. 2). The proper diagnosis of GSI prior to surgery was assessed in only these patients. In one case $(25 \%)$, enterolithotomy was completed in one stage with cholecystectomy and closure of the fistula during acute sur-

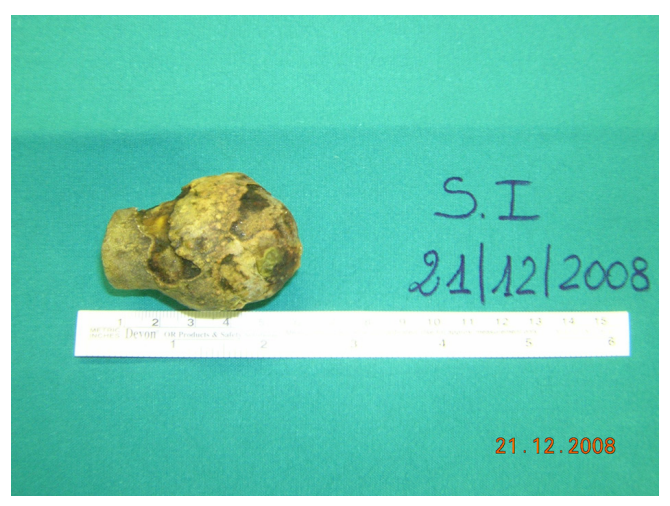

Figure 4. Gallstone extracted from the ileum. 
gery, while in 3 cases $(75 \%)$ enterolithotomy alone (without cholecystectomy and closure of the fistula) was performed (Fig. 3). The diameter of stone was equal or more than $4 \mathrm{~cm}$ in 3 case $(75 \%)$ (Fig. 4) and the location of the stone was jejunum in 3 cases $(75 \%)$ and ileum in $1(25 \%)$. The mean duration of hospitalization was 11.2 days (range 8 - 15). No patient showed further biliary symptoms after surgery. One patient (86-year-old women) died on the 14th postoperative day due to anaemia and melena complicated by myocardial failure. This patient was submitted to upper digestive endoscopy eight day after surgery to evaluate melena and no findings of bilioenteric fistula was found in the duodenum and first $20 \mathrm{~cm}$ of jejunum.

\section{Discussion}

The typical patient with GSI is female, elderly, with concomitant medical diseases and high risk (ASA 3 - 4) as shown in our patients, all of female gender, with a mean age of 81.7 years (range 75 - 86 years) and ASA 3 - 4. The incidence of GSI reported in the literature is $1-3 \%$ of all mechanical intestinal obstruction and it should become more common as a result of increase the aging population. Actually the prevalence of cholelithiasis increases with advancing age. The overall frequency of gallstone disease in the elderly has been estimated to be between $14 \%$ and $27 \%$ [4]. Older adult patients have more complications as a result of cholelithiasis, specifically acute cholecystitis, cholangitis, gallbladder perforation, gangrene, emphysematous cholecystitis, gallstone ileus and choledocholithiasis. In our report if we consider all mechanical intestinal obstruction the incidence is $0.66 \%$ ( 4 out of 601 cases), if instead we consider only small bowel obstruction (399 out of 601), the incidence increases to $1 \%$. However, we have considered elderly patients with small bowel obstruction only: the incidence is $2.01 \%$ in patients over the age of 65 (199 out of 601) and $3.73 \%$ in patients over the age of 80 ( 107 out of 601 ). These values are lower than the percentages reported in the literature where the prevalence is up to $25 \%$ in patients over 65 years of age with non-strangulated small bowel obstructions [3]. Instead, in cases of small bowel obstruction resulting in impacted foreign body we found an incidence of $36.36 \%$. In consideration of the site of stone impaction 3 types of intestinal obstruction are distinguished: high when the site of stone impaction is in the duodenum or in the jejunum, middle type when is in the ileum and low type when is in the colon [5]. The most frequent site of stone impaction is the ileum ( $>$ $60 \%$ of cases) and the gall stone is usually more than $2.5 \mathrm{~cm}$ in diameter $[3,6]$. Instead in our report the most frequent site is jejunum ( 3 out of 4 patients) and in 3 cases the diameter of gall stone was equal or more than $4 \mathrm{~cm}$.

The history, clinical, and radiologic findings are nonspecific, but indicative and about $50 \%$ of the patients have a history of gallbladder disease [7]. An early diagnostic can reduce the mortality rate. The symptoms may suggest a small bowel obstruction. Plain abdominal radiographs are often nonspecific and inconclusive revealing only signs of intestinal obstruction. At times the presence of concomitant pneumobilia may suggest the diagnosis, and only a decade ago, correct pre-operative diagnosis was as low as $20 \%$. However, recent advances in ultrasonography and computerised tomography can show often the Rigler's triad (pneumobilia, ectopic radio-opaque gallstone and intestinal distension) [8]. Presence of two of these radiological signs has been considered sufficient to establish a diagnostic. Nowadays the use of these imaging techniques, in combination with plain abdominal radiographs, can expedite the correct diagnosis in over $50 \%$ of cases decreasing preoperative delay [9]. Abdominal CT scans are the most important diagnostic tests due to their superior resolution and ability to identify a calculus in $78 \%$ of patients. This imaging technique has been reported to offer prompt and rapid pre-operative diagnosis of gallstone ileus with a sensitivity of $93 \%$ [10]. In addition it can show pneumobilia, intestinal obstruction and the thickness of the bile gallbladder's wall. Lassandro [11] in his report compared radiological findings in 27 patients: the Rigler's triad is observable on $14.81 \%$ of simple abdominal $\mathrm{x}$-rays, in $11.11 \%$ of abdominal sonograms and in $77.78 \%$ of abdominal CT scans.

Even though there is universal agreement on surgery as first-option treatment for these patients to relieve the intestinal obstruction by removing the stone, there is still reasonable disagreement about the surgical strategy because of low incidence of this disease. In fact, intestinal obstruction requires not a diagnostic approach but rather an emergency surgical treatment and prompt release of intestinal obstruction remains the cornerstone of management. Enterotomy and stone extraction will resolve the intestinal obstruction and it is quick, simple and fast. The disadvantages are the risk of further obstruction if there are residual stones within the gallbladder, persistent symptoms from an inflamed gallbladder and a possible increased risk of developing gallbladder cancer. For this reason single stage procedure with enterolithotomy, cholecystectomy and repair of biliary fistula is proposed to avoid these future risks. It ensures a definitive treatment, but there is a great disadvantage of high morbidity and mortality rate [3] due to the complexity of the intervention and the increased time of it. Considering complications related to the fistula are rare as most of them undergo spontaneous closure, only $10 \%$ of these patients will have postoperative biliary symptoms, the postoperative recurrence rate of gallstone ileus is $4.7 \%$, the mortality is $16.9 \%$ with a one-stage procedure, versus $11.7 \%$ following enterolithotomy (difference did not reach statistical significance: $\mathrm{P}<$ 0.17 ) [3], enterolithotomy alone remains the more performed operative method and considered to be appropriate in most patients. However, the one-stage-procedure is desirable if 
the condition of the patient allows it. Conservative therapy may also be considered for the treatment of GSI when the obstructing gallstone is smaller than $2 \mathrm{~cm}$ based on radiological measurement [12]. Instead, the two stage procedure (enterolithotomy initially followed at $4-6$ week by cholecystectomy and repair of the bilio-digestive fistula) should be performed only for symptomatic patients or patients developing complications during follow-up.

Based on these observations and the results of other published studies we believe to perform simple enterolithotomy as procedure of choice in GSI. Support for enterolithotomy alone results from it being the minimalist surgery possible in order to relieve bowel obstruction in the emergency situation. It is safe in both low and high-risk patients, requires a shorter operating time than the one stage procedure, and is technically less demanding. Instead, one stage procedure should be reserved only for highly selected patients with absolute indications like acute cholecystitis and/or gangrene of the gallbladder or in patients with greater life expectancy and less comorbidity and operative risk.

\section{Conflict of Interest}

None.

\section{Grant Support}

None.

\section{References}

1. Chou JW, Hsu CH, Liao KF, Lai HC, Cheng KS, Peng $\mathrm{CY}$, Yang MD, et al. Gallstone ileus: report of two cas- es and review of the literature. World J Gastroenterol. 2007;13(8):1295-1298.

2. Lobo DN, Jobling JC, Balfour TW. Gallstone ileus: diagnostic pitfalls and therapeutic successes. J Clin Gastroenterol. 2000;30(1):72-76.

3. Reisner RM, Cohen JR. Gallstone ileus: a review of 1001 reported cases. Am Surg. 1994;60(6):441-446.

4. Shah BB, Agrawal RM, Goldwasser B, Farah KF. Biliary diseases in the elderly. Pract Gastroenterol Sept 2008; 14-26.

5. Sfairi A, Farah A, Patel JC. [Computed tomography in the diagnosis of biliary ileus: a new case]. Gastroenterol Clin Biol. 1995;19(8-9):735-736.

6. Beuran M, Ivanov I, Venter MD. Gallstone ileus--clinical and therapeutic aspects. J Med Life. 2010;3(4):365371.

7. Clavien PA, Richon J, Burgan S, Rohner A. Gallstone ileus. Br J Surg. 1990;77(7):737-742.

8. Rigler LG, Borman CM, Noble JF. Gallstone obstruction: pathogenesis and roentgen manifestation. JAMA 1941; 117: 1753-68.

9. Riaz N, Khan MR, Tayeb M. Gallstone ileus: retrospective review of a single centre's experience using two surgical procedures. Singapore Med J. 2008;49(8):624626.

10. Yu CY, Lin CC, Shyu RY, Hsieh CB, Wu HS, Tyan YS, Hwang JI, et al. Value of CT in the diagnosis and management of gallstone ileus. World J Gastroenterol. 2005;11(14):2142-2147.

11. Lassandro F, Gagliardi N, Scuderi M, Pinto A, Gatta G, Mazzeo R. Gallstone ileus analysis of radiological findings in 27 patients. Eur J Radiol. 2004;50(1):23-29.

12. Nakao A, Okamoto Y, Sunami M, Fujita T, Tsuji T. The oldest patient with gallstone ileus: report of a case and review of 176 cases in Japan. Kurume Med J. 2008;55(12):29-33. 